\title{
Clinical Interpretation of Quantitative Sensory Testing as a Measure of Pain Sensitivity in Patients with Sickle Cell Disease
}

\author{
Amanda M. Brandow, DO, MS ${ }^{1,2,3}$ and Julie A. Panepinto, MD, MSPH ${ }^{1,2,3}$ \\ ${ }^{1}$ Section of Pediatric Hematology/Oncology, Milwaukee, WI, United States \\ ${ }^{2}$ Medical College of Wisconsin, Milwaukee, WI, United States \\ ${ }^{3}$ Children's Research Institute of the Children's Hospital of Wisconsin, Milwaukee, WI, United \\ States
}

\begin{abstract}
Patients with sickle cell disease (SCD) display significantly lower mean/median thermal and mechanical pain thresholds compared to controls. This suggests impaired pain sensitivity where stimuli produce exaggerated pain. Despite these mean/median differences, clinicians need to understand if patients meet criteria for impaired pain sensitivity. We defined thresholds for impaired cold, heat, and mechanical pain sensitivity in SCD patients. Using quantitative sensory testing (QST) we assessed cold, heat, and mechanical pain thresholds in SCD patients and African American controls $\geq 7$ years. Impaired pain sensitivity was defined as: 1) cold pain threshold one standard deviation (SD) above control median threshold; 2) heat pain threshold one SD below control median threshold; 3) mechanical pain threshold one SD below control median threshold. Fifty-five SCD patients and 57 controls participated. Impaired pain sensitivity thresholds were: 1) cold: $17.01^{\circ} \mathrm{C}, 2$ ) heat: $43.91^{\circ} \mathrm{C}, 3$ ) mechanical: $4.42 \mathrm{~g}$. Impaired cold pain sensitivity was the most common finding (63.6\%), then heat (60\%), and mechanical (38.2\%). Impaired pain sensitivity to $\geq 1$ testing modalities occurred in $81.8 \%$ of SCD patients. Determining impaired pain sensitivity thresholds increases clinical utility of QST. QST could be a screening tool to phenotype SCD pain, an outcome for pain interventional trials, or guide pain neurobiology investigations.
\end{abstract}

\section{Keywords}

sickle cell disease; pain sensitivity; neuropathic pain

\section{Introduction}

There are an increasing amount of data that peripheral and/or central nervous system abnormalities contribute to the development and maintenance of acute and chronic pain in

Corresponding Author: Amanda M. Brandow, DO, MS, 8701 Watertown Plank Road, Department of Pediatrics, Hematology/ Oncology, MFRC, Milwaukee, Wisconsin 53226, abrandow@ mcw.edu, Phone: 414-955-4170, Fax: 414-955-6543.

Conflicts of Interest: The authors declare no competing financial interests.

Authorship contributions

A.M.B. designed research, performed research, analyzed data, and wrote the manuscript and J.A.P. designed research and critically reviewed the manuscript. 
patients with sickle cell disease (SCD). Data in both the murine SCD model and patients with SCD reveal hypersensitivity to thermal and/or mechanical stimuli compared to healthy controls when assessed with quantitative sensory testing (QST). This suggests impaired pain sensitivity exists in SCD. ${ }^{1}$ QST involves a psychophysical evaluation of the somatosensory system. The premise of QST is that physical stimuli activate specific receptors and generate signals in the sensory nervous system. QST measures sensory loss (hyposensitivity) or gain (hypersensitivity) to stimuli (cold, heat, mechanical) that correlate with pain sensing somatic small nerve fibers. ${ }^{2,3}$ Impaired pain sensitivity on QST is present when a defined stimulus (cold, heat, mechanical) produces exaggerated pain in a patient compared to healthy controls and suggests pain processing abnormalities in the peripheral and/or central nervous system.

Existing studies of QST in patients with SCD report significant differences in mean/median thermal and mechanical pain thresholds between SCD patients and healthy controls. ${ }^{1,4-8}$ Reporting data in this manner is necessary and important, however, may not be the most meaningful for clinicians. For clinical purposes clinicians want to understand if an individual patient meets criteria for impaired pain sensitivity. This can be done by determining thresholds for impaired pain sensitivity as measured by QST. A defined threshold would allow a clinician to use this testing to perhaps recommend a different treatment or intervention making the testing more clinically meaningful and clinically useful. Determining whether a patient has impaired QST could also help guide further evaluation of the etiology of that patient's pain or serve as an outcome for interventions that could alter pain sensitivity. Thus, determining thresholds for impairment using QST measures is valuable. Furthermore, determining thresholds specifically for patients with SCD with proper race-matched controls is important as pain sensitivity is shown to vary based on ethnicity with African American people have increased sensitivity to pain as assessed by QST measures.9-11

To date, thresholds above or below which a patient is defined as having impaired pain sensitivity on QST have not been established in pediatric patients with SCD. Thus, in order to make QST data more clinically meaningful and interpretable, we sought to: 1) define thresholds for impaired cold, heat, and mechanical pain sensitivity in patients with SCD ages $\geq 7$ years and 2) determine the proportion of patients with SCD that meet criteria for impaired pain sensitivity with each testing modality. Our secondary objective was to compare age, gender and prior history of pain between patients with and without impaired pain sensitivity.

\section{Materials and Methods}

\section{Study Setting and Subjects}

This was a cross-sectional study completed between January 2010 and June 2011 at the Medical College of Wisconsin/Children's Hospital Wisconsin. The methods for this study were published in detail previously. ${ }^{4}$ Briefly, study patients included children and adults with SCD who were recruited from the Wisconsin Sickle Cell Center during outpatient clinic visits. Control subjects were African American and were siblings, parents/legal guardians, friends or relatives of patients with $\mathrm{SCD}$ and were also recruited through the clinic. All patients with SCD and controls were $\geq 7$ years of age. All subjects were excluded 
for the following: 1) chronic disease with associated pain manifestation other than SCD, 2) overt or silent stroke diagnosed on available neuroimaging, 3) analgesics used within 24 hours, 4) acute SCD pain event within 2 weeks.

The study was approved by the Institutional Review Board of the Children's Hospital of Wisconsin. Appropriate consent and assent (if applicable) were obtained. All subjects were given stipends for participation and for transportation to the testing site.

\section{Quantitative Sensory Testing (QST)}

All patients with SCD (in baseline health) and controls underwent QST that included assessment of cold, heat, and mechanical pain thresholds. The specific testing methodology used for QST is outlined in detail in our prior publication in patients with SCD. ${ }^{4}$ Briefly, cold and heat pain thresholds were assessed using the Medoc Thermal Sensory Analyzer (TSA-II) (Medoc, Israel) with a stimulus range between 0-32 ${ }^{\circ}$ Celsius. ${ }^{12}$ Mechanical pain thresholds were assessed using vonFrey monofilaments with a stimulus range between 0.026 and 110 grams. The thermal and mechanical pain thresholds were measured using the method of limits ${ }^{13}$ where subjects pushed a button (cold, heat) or spoke (mechanical) when the defined stimulus became painful. All testing was performed on the thenar eminence of the non-dominant hand in order to assess generalized pain sensitivity.

\section{Primary Outcome}

Our primary outcome was threshold for impaired pain sensitivity defined as: 1) cold pain threshold that was one standard deviation (SD) above median cold pain threshold in the control group; 2) heat pain threshold that was one SD below median heat pain threshold in the control group; 3) mechanical pain threshold that was one SD below median mechanical pain threshold in the control group. We adapted the methods published by Ezenwa et al, to define these thresholds. ${ }^{7}$ One SD was chosen to be consistent with other literature. ${ }^{14}$

\section{Covariates of interest}

Age, gender, and prior history of pain were covariates of interest due to their potential impact on our primary outcome. ${ }^{15-22}$ Priory history of pain was determined by the total number of emergency department visits or hospitalizations over the lifetime of the patient as recorded in our detailed comprehensive sickle cell clinical database, from the medical record, and/or from the patients' self-report.

\section{Statistical Analysis}

The cold, heat, and mechanical pain thresholds previously published were skewed. ${ }^{4}$ Thus, in order to determine the thresholds for impaired pain sensitivity bootstrap resampling was used to determine the standard deviation of the median. ${ }^{23}$ Thresholds for impaired pain sensitivity were then determined based on the criteria described above. The proportions of patients that had cold, heat, and mechanical pain thresholds above or below the calculated threshold for impaired pain sensitivity were determined. Mann-Whitney Test and Pearson Chi-square were used to compare age, gender, and prior history of pain (total number of lifetime emergency department visits and/or hospitalizations) between those with and without impaired pain sensitivity. Statistical significance was set at a p-value of $<0.05$. All 
analyses were completed by SPSS version 14.0 for Windows (SPSS, Chicago, IL) and STATA version 11.2 (STATA, College Station, TX).

\section{Results}

\section{Study Population}

A total of 55 patients with SCD and 57 African American controls completed QST. The demographics of the study population are displayed in Table I.

\section{One-half to two-thirds of patients with SCD met criteria for impaired pain sensitivity}

Using the previously described criteria for impaired pain sensitivity of 1 standard deviation from the median pain threshold in the control group, Table II displays the values above which patients meet criteria for being impaired in their cold pain sensitivity and below which patients meet criteria for impaired heat and mechanical pain sensitivity. Using these cutoff values we found that about two-thirds of patients with SCD had cold and heat pain thresholds that met criteria for impaired pain sensitivity and almost half of patients with SCD met criteria for impaired mechanical pain sensitivity (Table II). In order to give clinicians an understanding of where patients clustered above and below these thresholds for impairment, Figure 1 displays the spread of individual patient data above and below these thresholds.

\section{Almost a quarter of patients with SCD had impaired pain sensitivity with all modalities tested (cold, heat and mechanical)}

The number of patients that had impaired pain sensitivity in multiple testing modalities is displayed in Figure 2. About 22\% of patients had impaired pain sensitivity with all 3 testing modalities (cold, heat, and mechanical) and the majority $(81.8 \%, \mathrm{n}=45)$ had evidence of at least 1 pain sensitivity abnormality. Less than $20 \%$ had no hypersensitivity to any of the testing modalities.

\section{No differences in clinical characteristics between those with and without evidence of impaired pain sensitivity}

When characteristics were compared between those with one or more impairments in pain sensitivity and no impairments in pain sensitivity, there was no difference in median age [15 (IQR 10.5-19) vs. 13.5 yrs (IQR 11-21.5), p=0.939], gender [60\% female in both groups], or

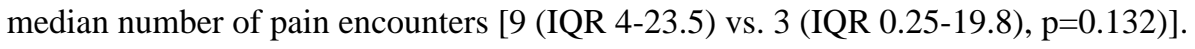

Furthermore, when clinical characteristics were compared between those with impaired pain sensitivity in all 3 testing modalities (cold, heat, mechanical) and no impairments in pain sensitivity there was no difference in median age [11.5 (IQR 7.25-16.25) vs. $13.5 \mathrm{yrs}$ (IQR 11-21.5), $\mathrm{p}=0.21$ ], gender [ $60 \%$ female vs. $60 \%$ female, $\mathrm{p}=0.69$ ], or median number of pain encounters [6.5 (IQR 3-21) vs. 3 (IQR 0.25-19.8), p=0.44)].

\section{Discussion}

In this study, we found that impaired cold pain sensitivity was not only the most common finding but that almost two-thirds of all patients with SCD met criteria for impaired cold 
pain sensitivity. These data provide supporting psychophysical evidence that corroborates the epidemiological data that increased numbers of pain events occur during colder temperatures and in fall and winter months compared to spring and summer months. ${ }^{24,25}$ The reason for this cold sensitivity continues to be unknown and importantly should continue to be an active area of investigation. We also found that the majority of patients (82\%) had evidence for impaired pain sensitivity in at least one testing modality providing additional evidence for the existence of peripheral and/or central sensitization in patients with SCD.

One prior study by Ezenwa et $\mathrm{al}^{7}$ in adults with SCD applied the threshold principals to the analysis of QST data. These investigators defined an abnormal thermal pain threshold as a cold/heat pain threshold that was $1 / 2$ standard deviation above or below the cold and heat pain thresholds of their historical control group. They found $72 \%$ of patients had abnormal cold pain sensitivity, $76 \%$ of patients had abnormal heat pain sensitivity, and $44 \%$ of subjects had abnormal mechanical pain sensitivity. ${ }^{7}$ Our study is unique in that it includes pediatric patients, a larger cohort, and African American controls. This inclusion of race-matched controls is very important in the study of pain sensitivity as pain sensitivity varies based on ethnicity. Specifically, African Americans display increased sensitivity to QST measures. ${ }^{9,11}$ In addition, unlike the Ezenwa study, we used one full standard deviation from the control median to determine our thresholds to be more conservative and to be consistent with other literature. ${ }^{14}$

In addition to the majority of patients displaying evidence of impaired pain sensitivity in at least one testing modality, we also found almost a quarter of patients were impaired in all 3 modalities tested (cold, heat, and mechanical impaired pain sensitivity). It is possible that these patients may represent a more severe cohort of patients that may be at risk for the development of chronic pain. Importantly, less than $20 \%$ of patients had no evidence of impaired pain sensitivity, thus these patients may represent a less severe cohort of patients with SCD in regards to pain. These data raise the question of whether the modality that is impaired (cold, heat, mechanical) provides clues to the underlying neurobiology that may result in the variation in pain expression that is seen from patient to patient. As QST continues to be used to phenotypically characterize the variability in pain sensitivity and expression in patients with SCD, opportunities to therapeutically target specific cellular and neurobiological processes may arise and should be considered when treating patients with pain. $^{26-28}$

We did not find differences in the age, gender, or prior history of pain in patients that had one or more impairments in pain sensitivity. This was contrary to our hypotheses, however, these were secondary analyses and thus the study is likely underpowered to find differences in these clinical characteristics. Furthermore, using total number of health care utilization encounters for pain defined as emergency department visits and hospitalizations for pain likely underestimates the amount of pain that patients experience. Data reveal that health care utilization only marks the tip of the iceberg of sickle cell pain as many pain events are managed at home and health care utilization is not sought. ${ }^{29}$ In addition, markers of pain hypersensitivity revealed by QST impairment may also reflect chronic pain and patients 
suffering from chronic pain may not be as likely to always seek medical care for treatment of their pain.

As additional QST research is conducted and further evidence from this contributes to the discovery of the neurobiological mechanisms for abnormal pain sensitivity in patients with $\mathrm{SCD}$, determining a cutoff for impaired pain sensitivity is clinically meaningful. Eventually, QST could become a screening test to further define a phenotypic cohort of patients with SCD whom meet criteria for impaired pain sensitivity. QST could help guide further evaluation of the etiology of an individual patient's pain, guide treatment decisions, or serve as an outcome for an intervention aimed at altering pain sensitivity. ${ }^{30-35}$ For example, if a patient is found to be impaired in their cold, heat, and/or mechanical pain sensitivity (i.e., display pain hypersensitivity) suggestive of neuropathic pain, this could make them eligible for a clinical trial for a neuropathic pain intervention and QST could be used as an outcome measure to reassess their response to the drug. This model has been used in clinical trials in pain related conditions other than SCD. ${ }^{31-33,35}$ Current pain assessment measures in SCD are primarily focused on pain intensity (numeric rating scale, visual analog scale, faces scale) and pain frequency. These measures do not focus on qualitative aspects of pain nor dissect phenotypic variation of pain. Assessing the phenotypic aspect of pain is important when investigating the underlying neurobiological etiology of pain. QST could be a novel way to screen for and follow pain in patients with SCD. In order to use QST in this way, the values measured need to be clinically meaningful and interpretable.

\section{Limitations}

Our study is limited by the cross-sectional nature of the data. The longitudinal variation of pain sensitivity thresholds is not known, thus it is difficult to interpret whether impaired pain sensitivity outcomes are the cause or the result of pain in patients with SCD. This study also does not assess whether there are further impairments in pain sensitivity that occur in relationship to a recent pain episode since we excluded patients that had an acute pain event within 2 weeks of testing. In this study, the impaired pain sensitivity found in baseline health suggests this impairment is likely to be permanent. In addition, since patients were tested in their baseline state of health we did not use factors such as pain intensity as a discriminatory factor. Furthermore, the analyses investigating the differences in covariates between patients with and without impaired pain sensitivity are likely underpowered. Thus, the conclusion that there are no differences in age, gender or prior history of pain between groups should be interpreted with caution. A larger study specifically powered to analyze these differences should be done to confirm or refute these findings. As previously discussed, the use of health care utilization as a marker of pain underestimates the true amount of pain patients experience outside the health care setting and thus pain severity and chronic pain are likely underrepresented in these data. ${ }^{29}$ The inclusion of pain diaries in future studies would facilitate the collection of data that could better assess frequent pain episodes managed at home and chronic pain. However, this was the best measure available that was not confounded by recall bias. The research staff that conducted QST were not blinded to whether the subject had SCD or was a control as those that conducted the testing are part of the primary SCD research staff. However, since the thermal testing is computer-driven this is less of a concern and mechanical testing followed testing procedures that have been 
standardized. Patients with SCD were excluded if there was a known history of overt or silent stroke. Screening MRI for all patients in the study was not feasible. Our study included family controls. Although this could have made our control group more similar to our patient group, this was a strength of the study. Inclusion of family controls allowed us to control for genetic and environmental factors that may influence pain sensitivity that are difficult to measure making them the ideal and necessary control group for the study. Finally, there are limitations of QST. The assessment of pain sensitivity outcomes could be affected by psychosocial functioning and determining this impact is part of an ongoing study. In addition, accuracy of QST depends on a well-established protocol that is consistent throughout the duration of the study. Our study utilized an established and consistent testing protocol and testing was administered by the same research staff throughout the duration of the study. Finally, QST alone is not able to determine which somatosensory pathway is disrupted along the neuroaxis. In other words, QST alone is not able to distinguish between whether peripheral and/or central nervous system abnormalities are contributing to the abnormal pain sensitization.

\section{Conclusions}

Determining a threshold for impaired pain sensitivity provides data that is clinically meaningful to health care providers. Using QST data, we established thresholds for impaired cold, heat and mechanical pain sensitivity. Based on these thresholds, almost a quarter of SCD patients were impaired in all 3 modalities tested and the majority was impaired in at least one modality. Impaired cold pain sensitivity was the most common finding supporting epidemiological data that increased numbers of pain events are associated with colder temperatures. Eventually, QST could serve as a screening tool to phenotype SCD pain, guide further evaluation of the etiology of pain, guide treatment decisions, or serve as an outcome for an intervention aimed at altering pain sensitivity.

\section{Acknowledgments}

We acknowledge Rebecca Farley, Robbie Kattappuram, Sylvia Torres, and Dawn Retherford for their assistance with testing and data collection. We also acknowledge Ray Hoffmann and Mahua Dasgupta for statistical support. We finally very importantly acknowledge all the patients, families, and control subjects that participated in the study.

Funding: This work was supported in part by grants from the National Institutes of Health National Heart, Lung, and Blood Institute 1K23 HL114636-01A1 (AMB) and U54 HL090503 (AMB), the American Society of Hematology (ASH) and the Midwest Athletes Against Childhood Cancer and Blood Diseases Fund (AMB).

\section{References}

1. Brandow AM, Farley RA, Panepinto JA. Early insights into the neurobiology of pain in sickle cell disease: A systematic review of the literature. Pediatr Blood Cancer. Sep; 2015 62(9):1501-1511. [PubMed: 25976161]

2. Arendt-Nielsen L, Curatolo M, Drewes A. Human experimental pain models in drug development: translational pain research. Curr Opin Investig Drugs. Jan; 2007 8(1):41-53.

3. Backonja MM, Walk D, Edwards RR, et al. Quantitative sensory testing in measurement of neuropathic pain phenomena and other sensory abnormalities. Clin J Pain. Sep; 2009 25(7):641647. [PubMed: 19692807] 
4. Brandow AM, Stucky CL, Hillery CA, Hoffmann RG, Panepinto JA. Patients with sickle cell disease have increased sensitivity to cold and heat. Am J Hematol. Jan; 2013 88(1):37-43. [PubMed: 23115062]

5. O'Leary JD, Crawford MW, Odame I, Shorten GD, McGrath PA. Thermal Pain and Sensory Processing in Children With Sickle Cell Disease. Clin J Pain. Apr 25.2013

6. Jacob E, Chan VW, Hodge C, Zeltzer L, Zurakowski D, Sethna NF. Sensory and Thermal Quantitative Testing in Children With Sickle Cell Disease. J Pediatr Hematol Oncol. Jul 10.2014

7. Ezenwa MO, Molokie RE, Wang ZJ, et al. Safety and Utility of Quantitative Sensory Testing among Adults with Sickle Cell Disease: Indicators of Neuropathic Pain? Pain practice : the official journal of World Institute of Pain. Jan 12.2015

8. Hollins M, Stonerock GL, Kisaalita NR, Jones S, Orringer E, Gil KM. Detecting the emergence of chronic pain in sickle cell disease. J Pain Symptom Manage. Jun; 2012 43(6):1082-1093. [PubMed: 22579409]

9. Edwards RR, Fillingim RB. Ethnic differences in thermal pain responses. Psychosom Med. MayJun;1999 61(3):346-354. [PubMed: 10367615]

10. Edwards CL, Fillingim RB, Keefe F. Race, ethnicity and pain. Pain. Nov; 2001 94(2):133-137. [PubMed: 11690726]

11. Campbell CM, Edwards RR, Fillingim RB. Ethnic differences in responses to multiple experimental pain stimuli. Pain. Jan; 2005 113(1-2):20-26. [PubMed: 15621360]

12. [September 6, 2011] Medoc TSA-II NeuroSensory Analyzer. http://www.medocweb.com/ medoc_en_tsaII_analyzer.aspx. Available at.

13. Meier PM, Berde CB, DiCanzio J, Zurakowski D, Sethna NF. Quantitative assessment of cutaneous thermal and vibration sensation and thermal pain detection thresholds in healthy children and adolescents. Muscle Nerve. Oct; 2001 24(10):1339-1345. [PubMed: 11562914]

14. Varni JW, Burwinkle TM, Seid M, Skarr D. The PedsQL 4.0 as a pediatric population health measure: feasibility, reliability, and validity. Ambulatory pediatrics : the official journal of the Ambulatory Pediatric Association. Nov-Dec;2003 3(6):329-341. [PubMed: 14616041]

15. Sethna NF, Meier PM, Zurakowski D, Berde CB. Cutaneous sensory abnormalities in children and adolescents with complex regional pain syndromes. Pain. Sep; 2007 131(1-2):153-161. [PubMed: 17329025]

16. Riley JL 3rd, Robinson ME, Wise EA, Myers CD, Fillingim RB. Sex differences in the perception of noxious experimental stimuli: a meta-analysis. Pain. Feb; 1998 74(2-3):181-187. [PubMed: 9520232]

17. Fillingim RB, King CD, Ribeiro-Dasilva MC, Rahim-Williams B, Riley JL 3rd. Sex, gender, and pain: a review of recent clinical and experimental findings. J Pain. May; 2009 10(5):447-485. [PubMed: 19411059]

18. Edwards RR, Fillingim RB. Age-associated differences in responses to noxious stimuli. J Gerontol A Biol Sci Med Sci. Mar; 2001 56(3):M180-185. [PubMed: 11253160]

19. Edwards RR, Fillingim RB. Effects of age on temporal summation and habituation of thermal pain: clinical relevance in healthy older and younger adults. J Pain. Dec; 2001 2(6):307-317. [PubMed: 14622810]

20. Zohsel K, Hohmeister J, Flor H, Hermann C. Somatic pain sensitivity in children with recurrent abdominal pain. Am J Gastroenterol. Jun; 2008 103(6):1517-1523. [PubMed: 18510619]

21. Zohsel K, Hohmeister J, Oelkers-Ax R, Flor H, Hermann C. Quantitative sensory testing in children with migraine: preliminary evidence for enhanced sensitivity to painful stimuli especially in girls. Pain. Jul; 2006 123(1-2):10-18. [PubMed: 16495010]

22. Hogeweg JA, Kuis W, Huygen AC, et al. The pain threshold in juvenile chronic arthritis. Br J Rheumatol. Jan; 1995 34(1):61-67. [PubMed: 7881842]

23. Manly, BFJ. Randomization, Bootstrap and Monte Carlo Methods in Biology. Third Edition. Chapman \& Hall; New York: 1997.

24. Smith WR, Bauserman RL, Ballas SK, et al. Climatic and geographic temporal patterns of pain in the Multicenter Study of Hydroxyurea. Pain. Nov; 2009 146(1-2):91-98. [PubMed: 19683393]

25. Resar LM, Oski FA. Cold water exposure and vaso-occlusive crises in sickle cell anemia. J Pediatr. Mar; 1991 118(3):407-409. [PubMed: 1999780] 
26. Nozadze I, Tsiklauri N, Gurtskaia G, Tsagareli MG. Role of thermo TRPA1 and TRPV1 channels in heat, cold, and mechanical nociception of rats. Behavioural pharmacology. Aug 13.2015

27. Hucho T, Levine JD. Signaling pathways in sensitization: toward a nociceptor cell biology. Neuron. Aug 2; 2007 55(3):365-376. [PubMed: 17678851]

28. Levine JD, Alessandri-Haber N. TRP channels: targets for the relief of pain. Biochim Biophys Acta. Aug; 2007 1772(8):989-1003. [PubMed: 17321113]

29. Smith WR, Penberthy LT, Bovbjerg VE, et al. Daily assessment of pain in adults with sickle cell disease. Ann Intern Med. Jan 15; 2008 148(2):94-101. [PubMed: 18195334]

30. Arendt-Nielsen L. Central sensitization in humans: assessment and pharmacology. Handbook of experimental pharmacology. 2015; 227:79-102. [PubMed: 25846615]

31. de Hoogd S, Ahlers SJ, van Dongen EP, Tibboel D, Dahan A, Knibbe CA. Remifentanil versus fentanyl during cardiac surgery on the incidence of chronic thoracic pain (REFLECT): study protocol for a randomized controlled trial. Trials. 2014; 15:466. [PubMed: 25429720]

32. Breuer AJ, Mainka T, Hansel N, Maier C, Krumova EK. Short-term treatment with parecoxib for complex regional pain syndrome: a randomized, placebo-controlled double-blind trial. Pain physician. Mar-Apr;2014 17(2):127-137. [PubMed: 24658473]

33. Stefani LC, Muller S, Torres IL, et al. A Phase II, Randomized, Double-Blind, Placebo Controlled, Dose-Response Trial of the Melatonin Effect on the Pain Threshold of Healthy Subjects. PLoS One. 2013; 8(10):e74107. [PubMed: 25947930]

34. Kroigard T, Schroder HD, Qvortrup C, et al. Characterization and diagnostic evaluation of chronic polyneuropathies induced by oxaliplatin and docetaxel comparing skin biopsy to quantitative sensory testing and nerve conduction studies. European journal of neurology : the official journal of the European Federation of Neurological Societies. Apr; 2014 21(4):623-629.

35. Bouwense SA, Olesen SS, Drewes AM, Poley JW, van Goor H, Wilder-Smith OH. Effects of pregabalin on central sensitization in patients with chronic pancreatitis in a randomized, controlled trial. PLoS One. 2012; 7(8):e42096. [PubMed: 22879908] 

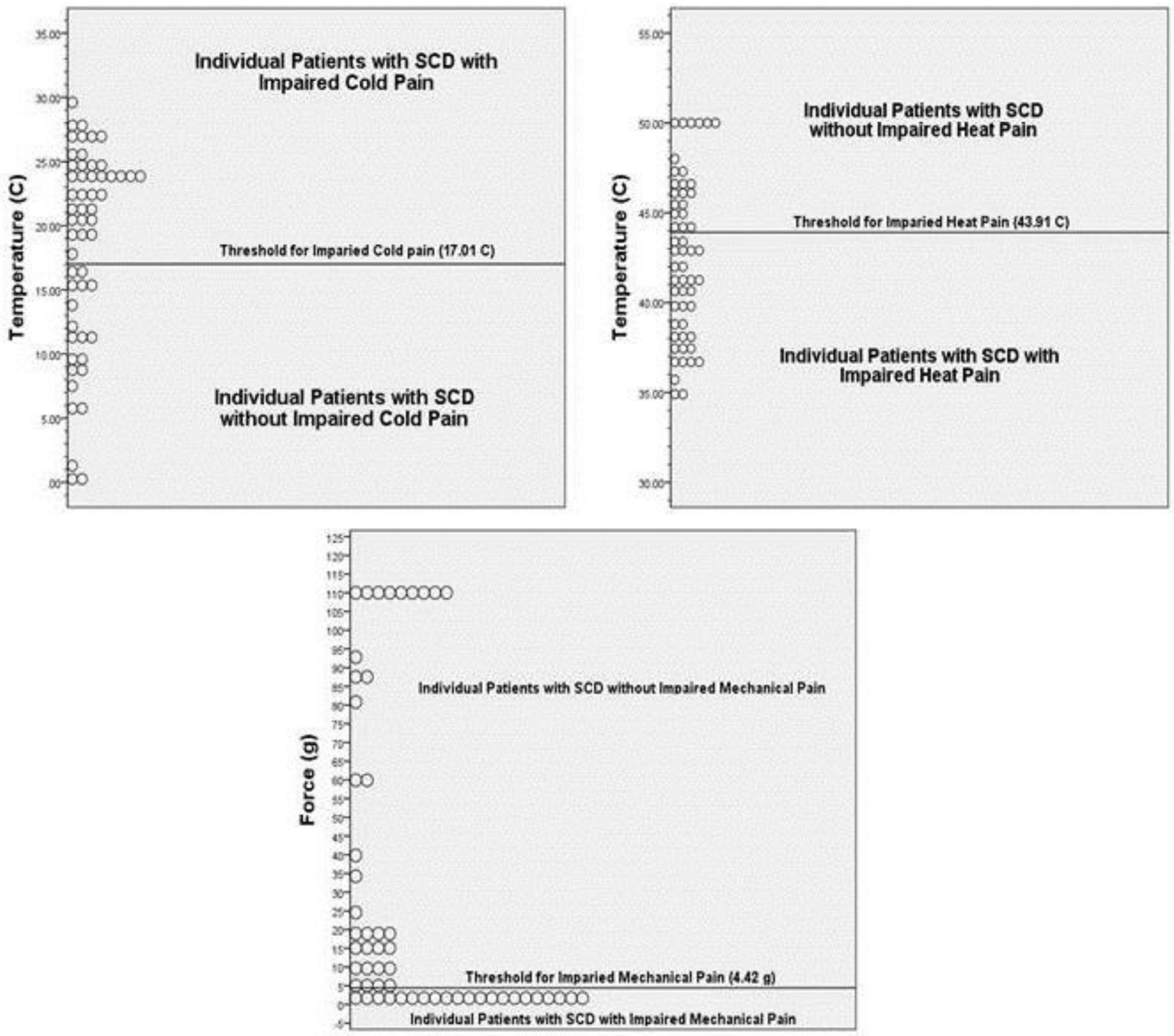

Figure 1.

Pictorial description of the spread of individual sickle cell disease patient pain thresholds that were in the impaired or non-impaired range for cold, heat, and mechanical pain 


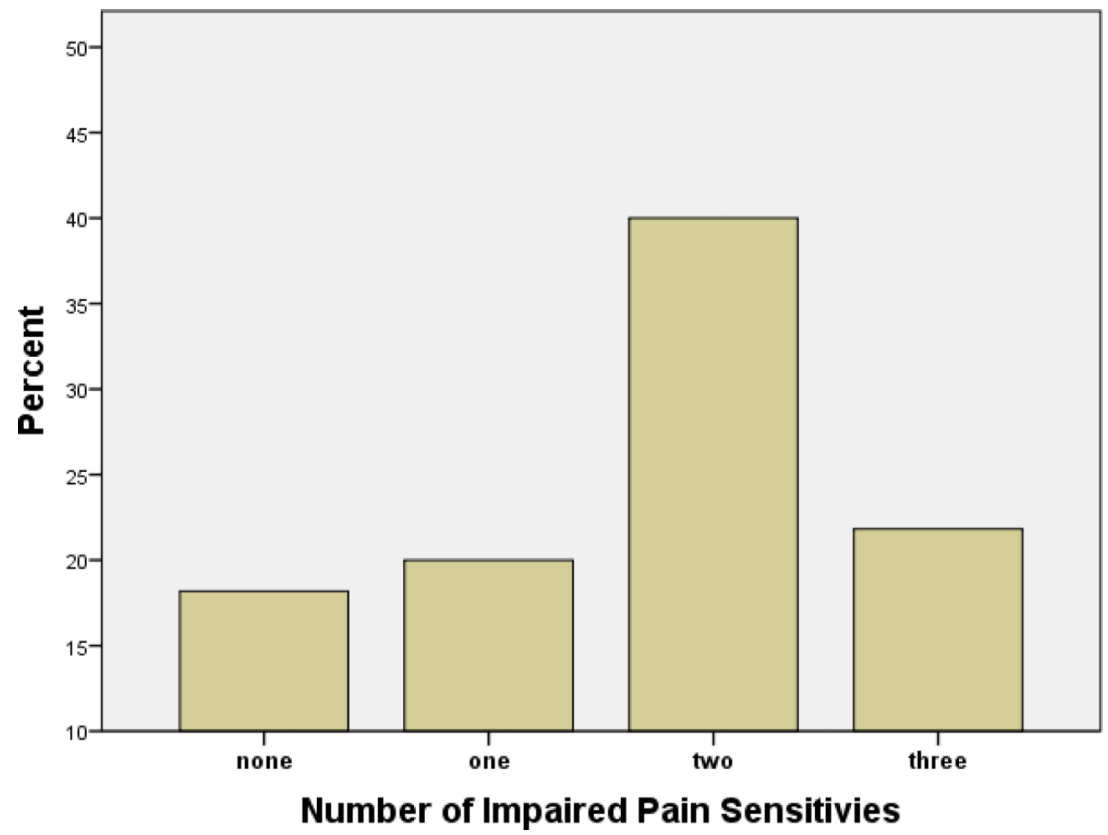

Figure 2.

Proportion of patients with sickle cell disease with varying numbers of impaired pain sensitivities 
Table I

Demographics and Baseline Characteristics of Patients with SCD and Controls

\begin{tabular}{|c|c|c|c|}
\hline Variable & SCD Patients $(n=55)$ & Controls $(n=57)$ & p-value \\
\hline \multicolumn{4}{|l|}{ Age (years) } \\
\hline Mean $( \pm \mathrm{SD})$ & $15.4( \pm 6.3)$ & $16.3( \pm 10.2)$ & 0.59 \\
\hline Median (IQR) & $14(11-19)$ & $12(9-18)$ & 0.42 \\
\hline \multicolumn{4}{|l|}{ Gender } \\
\hline Female & $33(60 \%)$ & $32(56 \%)$ & 0.68 \\
\hline Sickle Cell Genotype & & N/A & N/A \\
\hline $\mathrm{HbSS}$ & $37(67 \%)$ & & \\
\hline $\mathrm{HbSC}$ & $10(18 \%)$ & & \\
\hline $\mathrm{HbS} \beta+$ thal & $6(11 \%)$ & & \\
\hline Other & $2(4 \%)$ & & \\
\hline Trait Status & N/A & & N/A \\
\hline Unknown & & $5(9 \%)$ & \\
\hline No & & $28(49 \%)$ & \\
\hline$\underline{\text { Yes }}$ & & $\underline{24(42 \%)}$ & \\
\hline$S$ trait & & $16(28 \%)$ & \\
\hline $\mathrm{C}$ trait & & $2(4 \%)$ & \\
\hline Thal trait & & $1(2 \%)$ & \\
\hline Unknown & & $5(9 \%)$ & \\
\hline $\begin{array}{l}\text { Hydroxyurea } \\
\text { Yes }\end{array}$ & $23(42 \%)$ & N/A & \\
\hline Number of Prior Pain Encounters & & N/A & N/A \\
\hline Median (IQR) & $8(3.5-23.5)$ & & \\
\hline
\end{tabular}


Table II

Thresholds for Impaired Pain Sensitivity and Proportion of Patients with SCD with Impaired Pain Sensitivity $(\mathrm{n}=55)$

\begin{tabular}{|l|c|c|}
\hline & Threshold for Impairment $^{\#}$ & Proportion with Impaired Pain Sensitivity $^{*}$ \\
\hline Cold Pain Threshold & $>17.01^{\circ} \mathrm{C}$ & $63.6 \%(\mathrm{n}=35)$ \\
\hline Heat Pain Threshold & $<43.91^{\circ} \mathrm{C}$ & $60 \%(\mathrm{n}=33)$ \\
\hline Mechanical Pain Threshold & $<4.42 \mathrm{~g}$ & $38.2 \%(\mathrm{n}=21)$ \\
\hline
\end{tabular}

${ }^{\circ} \mathrm{C}=$ degrees Celsius, $\mathrm{g}=$ grams

\#

SD from control median

proportion of patients with SCD that had pain sensitivity thresholds that exceeded 1 SD from the control median pain sensitivity thresholds 